\title{
La Geometría de la Naturaleza: Benoit Mandelbrot
}

\author{
Vernor Arguedas T. \\ vernor.arguedas@ucr.ac.cr \\ Escuela de Matemática \\ Universidad de Costa Rica
}

Las nubes no son esferas, las montañas no son conos, las costas no son círculos, y las cortezas de los árboles no son lisas, ni los relámpagos viajan en una línea recta. Mandelbrot, de su libro "Introduction to The Fractal Geometry of Nature"

Este matemático polaco francés norteamericano gozó siempre de una gran reputación, que se acrecentó con el redescubrimiento de conceptos que condujeron a la dimensión fractal y a los fractales. ¿Existe una matemática que modela de manera acertada a ciertos procesos de la naturaleza? ¿Es sencilla esta matemática?



1924-2010

La matemática siempre ha servido de lenguaje de las ciencias, sobre todo de las llamadas por algunos fácticas. A partir de ellas se han construido modelos que simulan la realidad con mayor o menor éxito y que frecuentemente estos modelos sustituyen a la misma realidad. En el caso de los procesos "rugosos" -roughness en el lenguaje de Mandelbrot- tales como pliegues, o bien fenómenos que se autoreplican como los proceso de reproducción de células, las matemáticas -salvo modelos estadísticos y algunos analíticos-han tenido un éxito escaso. Volviendo a Mandelbrot hay muchísimas referencias en línea acerca de su biografía y su obra como por ejemplo: http://es.wikipedia.org/wiki/Benot_Mandelbrot

El siglo XXI nos da la oportunidad de conocer a algunas de estas grandes figuras, cómo eran físicamente, cómo explicaban sus teorías, en el caso de Mandelbrot podemos ver en un video de 17 minutos al maestro explicando a una audiencia general sus conceptos, quienes lo vean pueden escoger subtítulos en diversas lenguas, castellano incluido (http://www.ted.com/talks/benoit_mandelbrot_fractals_ the_art_of_roughness.html).

El video se filmó en febrero del 2010, pocos meses antes de su deceso, y me parece muy educativo para los lectores de este artículo que vean a quien fue una leyenda viviente en el mundo de las matemáticas. Con simpatía y sencillez explica los diversos conceptos que él desarrolló y cómo fue que empezó a estudiar los conjuntos de Julia a instancias de su tío matemático. Él reconoce de manera simple que sin las computadoras no hubiera podido avanzar mucho. Así que los fractales también son un producto de los avances en tecnología computacional.

Para quien desea aprender algunos detalles de los fractales, la referencia: http://es.wikipedia.org/ wiki/Fractal es útil, se presentan algunos aspectos de la teoría de fractales explicados de manera aceptable. Quienes desean profundizar más pueden consultar en: http://matap.dmae.upm.es/cursofractales/ 
index.htm se trata de un curso en línea sobre fractales.

Hay muchos programas para dibujar fractales sencillos, en el sitio de Mathematica: http://demonstrations. wolfram.com/JuliaSetsAndTheMandelbrotSet/ se puede ver una demostración con Mathematica e incluso bajar el código fuente.

La teoría de los fractales y el concepto de dimensión de Haussdorf no es sencilla, de ninguna manera va de acuerdo a lo que predica la navaja de Ockham, que no es más que una consecuencia del idealismo platónico y de la creencia de que la naturaleza es sencilla.

El principio es atribuido al fraile franciscano inglés del siglo XIV Guillermo de Ockham que forma la base del reduccionismo metodológico. Este principio ya formaba parte de la filosofía medieval aunque fue Ockham quien lo utilizó de forma filosófica. Sin embargo, no solamente es un principio metodológico sino que, además, tiene características gnoseológicas y ontológicas.

\section{Pluralitas non est ponenda sine necessitate (la pluralidad no se debe postular sin necesidad.)}

En su forma más simple, el principio de Ockham indica que las explicaciones nunca deben multiplicar las causas sin necesidad. El divulgador científico Arthur Clark presentó un programa titulado: Fractals -The Colors of Infinity-, el cual se encuentra en youtube.com en seis partes cortas.

La primera parte se encuentra en http://www.youtube.com/watch?v=qB8m85p7GsU, está en idioma inglés. Una versión con subtítulos en castellano se encuentra en: http://www . youtube . com/watch?v= 9212HsKuoGk, tenemos que agradecerle al profesor Rafael Miranda Molina este esfuerzo de subtitular en 4 partes el programa de Clark. Por cierto su canal Geometría Dinámica tiene materiales muy valiosos. Lo mismo se debe decir de su blog: http://www.geometriadinamica.cl

El concepto de dimensión tiene múltiples significados en matemáticas, por ejemplo en álgebra lineal significa el número máximo de vectores linealmente independientes de un espacio vectorial. En teoría de la medida, la dimensión de Haussdorf tiene un sentido diferente, en el curso en línea que citamos sobre fractales, http://matap.dmae.upm.es/cursofractales/index.htm, de los profesores Bartolo Luque y Aida Agea, transcribimos una explicación de varias definiciones usadas en geometría fractal:

He aquí la cita textual del capítulo 3 del curso en red:

\section{Midiendo longitudes y volúmenes}

Una forma de medir la longitud de una curva es aproximarla a la longitud de una serie de pequeñas rectas que la recubren. A ese procedimiento los matemáticos lo llaman rectificación. Cuanto más pequeñas sean las rectas escogidas para el recubrimiento, más exacta será nuestra medida.

Pero...¿qué ocurre si intentamos medir la "longitud total" de un cuadrado? No su perímetro, sino la longitud del cuadrado mismo por este método de rectificación.¿Tiene, siquiera, sentido tal pregunta?

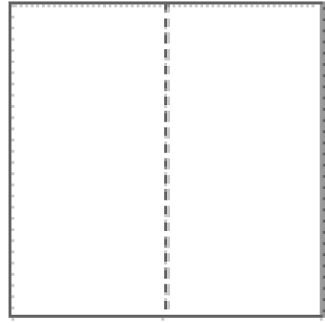

Longitud estimada $=1 \times 1$

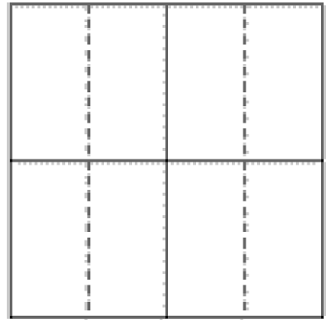

Longitud estimada $=4 \times 1 / 2$

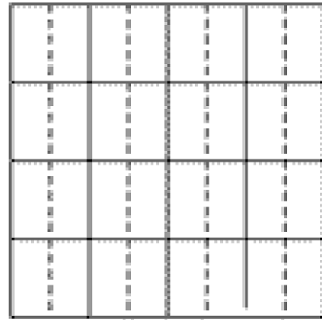

Longitud estimada $=16 \times 1 / 4$ 
Intentemos "rectificar" el cuadrado de lado $L=1$ de la figura. Para ello, probamos a recubrirlo con una línea. No hay duda de que es un recubrimiento inicial bastante burdo, pero así podremos hacer el procedimiento sistemático. Nuestro valor inicial de "longitud de un cuadrado" es $L_{1}=1$. Dividamos el cuadrado en cuatro cuadrados idénticos y tracemos 4 líneas que intenten recubrir cada uno de estos cuatro cuadrados. Cada una de estas líneas medirá $\frac{1}{2}$. La nueva medida $L_{2}$ será $L_{2}=4 \frac{1}{2}=2$. Una vez hemos definido el procedimiento, iteremos: ahora tenemos 16 cuadrados y 16 líneas de longitud $1 / 4$, en total $L_{3}=16 \frac{1}{4}=4$. En el paso cuatro tendremos $L_{4}=64 \frac{1}{8}=8$. Y así sucesivamente. Cuando hayamos repetido esta tediosa, pero expeditiva operación infinitas veces, podremos decir que hemos recubierto el cuadrado con líneas. No existirá ni un solo punto por el que no pase una línea, ni por ninguno de ellos pasará a la vez más de una. Para hallar matemáticamente el valor de la longitud de la línea que recubre al cuadrado empleamos el límite:

$$
L=\lim _{n \rightarrow \infty} 2^{2 n}\left(\frac{1}{2^{n}}\right)=\lim _{n \rightarrow \infty} 2^{n}=\infty
$$

La rara pregunta: "Cuál es la longitud de un cuadrado?" puede entonces responderse. Su longitud es infinita. Intentemos responder ahora a:¿Cuál es el volumen de un cuadrado? Para medir el volumen de un objeto geométrico, normalmente, hallamos el límite de la suma de elementos de volumen infinitesimal que lo rellenan. Apliquemos ese procedimiento intuitivamente claro para figuras con volumen, al caso de un cuadrado. Necesitamos recubrir el cuadrado con pequeños volúmenes que no dejen huecos, ni excedentes. Lo conseguiremos con un procedimiento exhaustivo semejante al anterior.
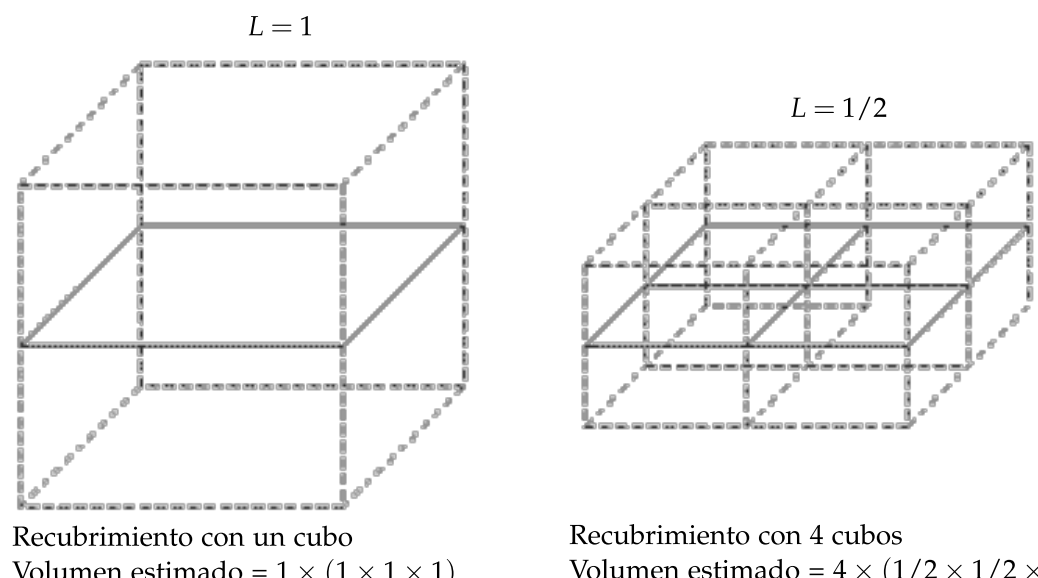

Recubrimiento con 4 cubos

Volumen estimado $=1 \times(1 \times 1 \times 1)$

Volumen estimado $=4 \times(1 / 2 \times 1 / 2 \times 1 / 2)$

Nuestra primera aproximación será de nuevo un recubrimiento burdo: una sola caja cúbica que contiene al cuadrado como sección transversal. Así, $V_{1}=1 \cdot 1 \cdot 1=1$. De nuevo, dividamos el cuadrado en cuatro pedazos idénticos y sobre cada uno repitamos el proceso anterior: recubrámoslos con cubos de arista correspondiente. Ahora tenemos 4 cubos de volumen $\frac{1}{2} \cdot \frac{1}{2} \cdot \frac{1}{2}=\frac{1}{8}$. La nueva aproximación será $V_{2}=4 \cdot\left(\frac{1}{2}\right)^{3}=\frac{1}{2}$. Si volvemos a dividir cada cuadrado en 4 trozos necesitaremos 16 cubos de tamaño $\left(\frac{1}{4}\right)^{3}$, cada uno para recubrir totalmente al cuadrado inicial. Así $V_{3}=16 \cdot\left(\frac{1}{4}\right)^{3}=\frac{1}{4}$. Repetimos y conseguimos: $V_{4}=64 \cdot\left(\frac{1}{8}\right)^{3}=\frac{1}{8}$. Si iteramos el proceso infinitamente, como hicimos antes, conseguiremos el volumen del cuadrado:

$$
V_{\infty}=\lim _{n \rightarrow \infty} 2^{2 n}\left(\frac{1}{2^{n}}\right)^{3}=\lim _{n \rightarrow \infty} \frac{1}{2^{n}}=0
$$

De modo que la longitud de un cuadrado es infinita y el volumen es cero. Intuitivamente vemos que el resultado tiene sentido: nos hacen falta infinitas líneas de longitud finita para recubrir un objeto plano, así que 
podemos decir que su longitud, en cierto sentido, es infinita. Además, el espesor de un objeto plano es nulo, de modo que su volumen también debería serlo. En realidad, este resultado obtenido es general: para cualquier objeto geométrico, medidas que usen dimensiones más bajas que su propia dimensión resultan infinitas y más altas, cero. Pero,¿qué podemos decir de medidas sobre objetos fractales?¿Seguirá funcionando este resultado general? Intentemos medir la longitud de un triángulo de Sierpinski. El recubrimiento a través de líneas nos viene sugerido de forma natural por la propia construcción del objeto geométrico.

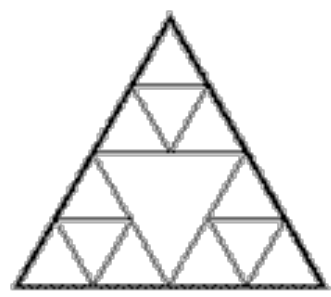

$L=3 \times 1=3$

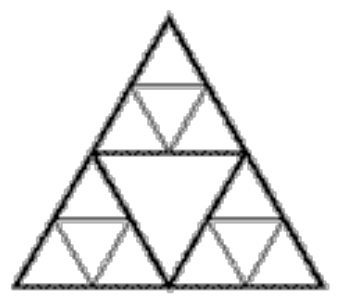

$L=3 \times 1+3 \times 1 / 2=4.5$

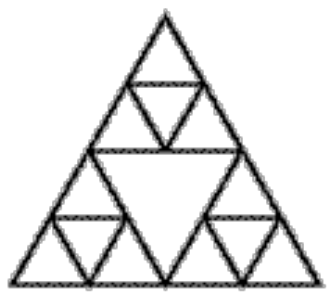

$L=3 \times 1+3 \times 1 / 2+9 \times 1 / 4=6.75$

Si la longitud del lado del triángulo equilátero inicial es 1, una primera aproximación de la longitud será $L_{1}=3 \cdot 1=3$ (en la figura aparecen en negrita los segmentos que usamos en nuestra suma). Si tomamos en cuenta además el triángulo central de lado $\frac{1}{2}$, añadimos a la longitud anterior $3 \frac{1}{2}$, y entonces: $L_{2}=3+3 \frac{1}{2}=4,5$. Si añadimos los siguientes nueve triángulos: $L_{3}=3+3 \frac{1}{2}+9 \frac{1}{4}=6,75$. El siguiente recubrimiento afina más aún, consiguiendo: $L_{4}=3+3 \frac{1}{2}+9 \frac{1}{4}+27 \frac{1}{8}=10,125$. Y en el límite, cuando recubramos todo el triángulo de Sierpinski, tendremos:

$$
L_{\infty}=3+\sum_{i=0}^{\infty}\left(\frac{3}{2}\right)^{i}
$$

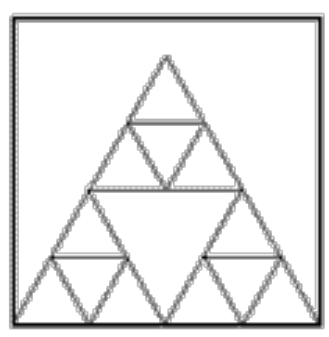

$S=1 \times 1=1$

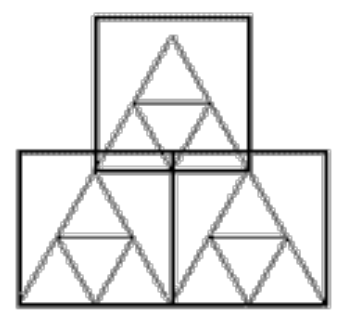

$S=3 \times(1 / 2 \times 1 / 2)=0.75$



$S=3 \times 3 \times(1 / 2 \times 1 / 4)=0.5625$

Calculemos el área. Emplearemos cuadrados para recubrir al triángulo. Nuestra primera aproximación emplea un cuadrado de lado 1 como muestra la figura. De modo que tenemos $S_{1}=1$. El siguiente paso afina un poco más el cálculo utilizando tres cuadrados de lado $\frac{1}{2}$. Eso nos da: $S_{2}=3\left(\frac{1}{2}\right)^{2}=0.75$. Seguidamente utilizamos 9 cuadrados de lado $\frac{1}{4}$, esto es, $S_{3}=9\left(\frac{1}{4}\right)^{2}=0,5625$. Ahora 27 cuadrados de lado un $1 /$ over 8 con área estimada de $S_{4}=27\left(\frac{1}{8}\right)^{2}=0,421875 \ldots$ Al llevar el proceso al límite conseguimos el área del triángulo de Sierpinski:

$$
S_{\infty}=\lim _{n \rightarrow \infty}\left[3^{n} \cdot\left(\frac{1}{2^{n}}\right)^{2}\right]=\lim _{n \rightarrow \infty}\left(\frac{3}{4}\right)^{n}=0
$$


Sorprendid@ ? El triángulo de Sierpinski es un objeto geométrico de infinita longitud, aunque se encuentra en una región finita del plano, cosa que implica dimensión mayor que uno. Pero a la vez tiene área nula, que indica dimensión menor que 2. Pero entonces, qué dimensión tiene? Intentaremos responder a la pregunta de cuál es la dimensión del triángulo de Sierpinski. Para ello vamos a aplicar el siguiente método a distintas figuras geométricas: las recubriremos exactamente con $\mathrm{N}$ piezas de tamaño característico $\mathrm{R}$ e intentaremos encontrar la relación funcional entre $\mathrm{N}$ y R. Sea un segmento de longitud $\mathrm{L}=1$. Podemos recubrirlo (Cuadro 1).

\begin{tabular}{|l|l|l|}
\hline 2 segmentos de tamaño $\frac{1}{2}$ & $N=2, R=\frac{1}{2}$ & $\left(\frac{1}{2}\right)^{-1}=2$ \\
\hline 4 segmentos de tamaño $\frac{1}{4}$ & $N=4, R=\frac{1}{4}$ & $\left(\frac{1}{4}\right)^{-1}=4$ \\
\hline 8 segmentos de tamaño $\frac{1}{8}$ & $N=8, R=\frac{1}{8}$ & $\left(\frac{1}{8}\right)^{-1}=8$ \\
\hline$\ldots$ & $\ldots$ & $\ldots$ \\
\hline $2^{r}$ segmentos de tamaño $\left(\frac{1}{2}\right)^{n}$ & $N=2^{n}, R=\frac{1}{2^{n}}$ & $R^{-1}=N$ \\
\hline
\end{tabular}

Observa que el exponente -1 cambiado de signo coincide con la dimensión 1 de una recta.
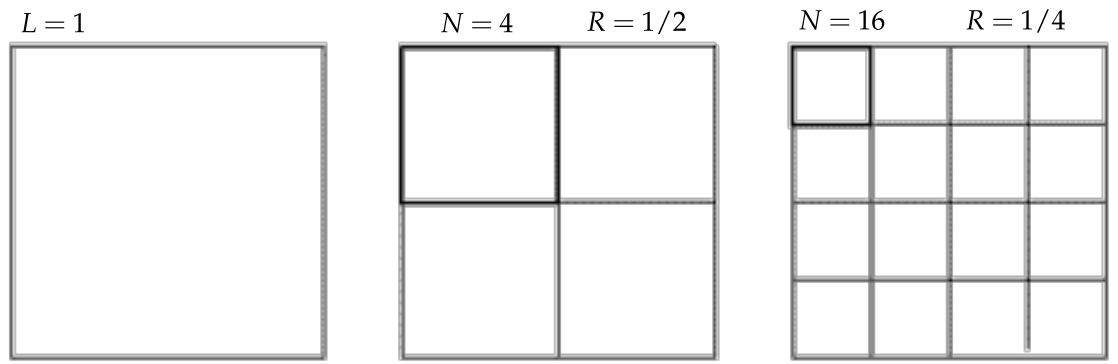

\begin{tabular}{|l|l|l|}
\hline 4 cuadrados de tamaño $\frac{1}{2}$ & $N=4, R=\frac{1}{2}$ & $\left(\frac{1}{2}\right)^{-2}=4$ \\
\hline 16 cuadrados de tamaño $\frac{1}{4}$ & $N=16, R=\frac{1}{4}$ & $\left(\frac{1}{4}\right)^{-2}=16$ \\
\hline 64 cuadrados de tamaño $\frac{1}{8}$ & $N=64, R=\frac{1}{8}$ & $\left(\frac{1}{8}\right)^{-2}=64$ \\
\hline$\ldots$ & $\ldots$ & $\ldots$ \\
\hline $2^{2 n}$ cuadrados de tamaño $\frac{1}{2^{n}}$ & $N=2^{2 n}, R=\frac{1}{2^{n}}$ & $R^{-2}=N$ \\
\hline
\end{tabular}

Ahora el exponente es -2, de nuevo cambiando el signo conseguimos la dimensión conocida. En este caso 2 

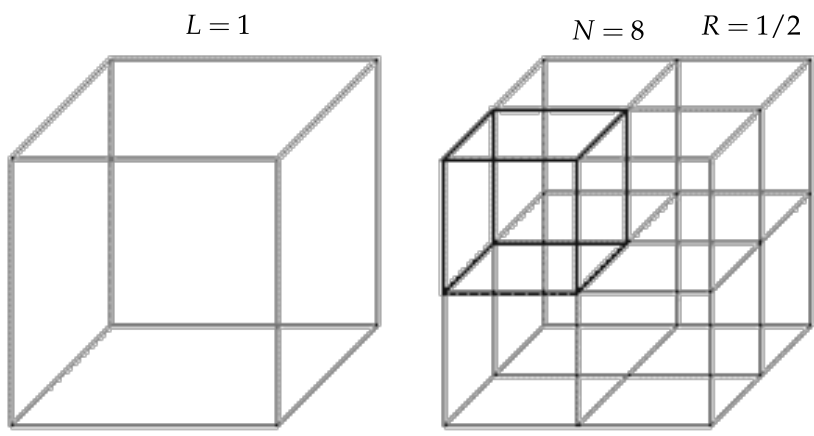

La cosa promete... Ahora tomemos un cubo. Sea un cubo de arista $\mathrm{L}=1$. Podemos recubrirlo, por ejemplo, con:

\begin{tabular}{|l|l|l|}
\hline 8 cubos de arista $\frac{1}{2}$ & $N=8, R=\frac{1}{2}$ & $\left(\frac{1}{2}\right)^{-3}=8$ \\
\hline 64 cubos de arista $\frac{1}{4}$ & $N=64, R=\frac{1}{4}$ & $\left(\frac{1}{4}\right)^{-3}=64$ \\
\hline 512 cubos de arista $\frac{1}{8}$ & $N=512, R=\frac{1}{8}$ & $\left(\frac{1}{8}\right)^{-3}=512$ \\
\hline$\ldots$ & $\ldots$ & $\cdots$ \\
\hline $2^{3 n}$ cubos de arista $\frac{1}{2^{n}}$ & $N=2^{3 n}, R=\frac{1}{2^{n}}$ & $R^{-3}=N$ \\
\hline
\end{tabular}

Y efectivamente el procedimiento nos devuelve dimensión 3. Así que parece que la siguiente regla nos determina la dimensión de un objeto geométrico: recubrimos exactamente con $\mathrm{N}$ piezas de tamaño característico R. La relación

$$
N=R^{D}
$$

nos determina la dimensión D del objeto geométrico. Qué exponente D encontramos al aplicar este método al triángulo de Sierpinski? Supongamos, sin pérdida de generalidad, que el lado del triángulo de Sierpinski mide 1. Podemos recubrir exactamente el triángulo de Sierpinski con copias reducidas. Por construcción sabemos que debemos emplear tres copias idénticas reducidas a $\frac{1}{3}$ del triángulo original. Cada uno de los tres triángulos idénticos debería tener lado $R=\frac{1}{2}$. Cada uno de estos triángulos a su vez está formado por tres copias reducidas, lo que hace un total de 9 copias $\operatorname{con} R=\frac{1}{4}$. Ahora ya vemos el proceso, el siguiente paso son 27 triángulos de lado $R=\frac{1}{8}$. Recordemos nuestra última conclusión sobre la dimensión: $N=R^{D}$. Recopilemos:

\begin{tabular}{|l|l|l|}
\hline 3 triángulos de lado $\frac{1}{2}$ & $N=3, R=\frac{1}{2}$ & $\left(\frac{1}{2}\right)^{-D}=3$ \\
\hline 9 triángulos de lado $\frac{1}{4}$ & $N=9, R=\frac{1}{4}$ & $\left(\frac{1}{4}\right)^{-D}=9$ \\
\hline 27 triángulos de lado $\frac{1}{8}$ & $N=27, R=\frac{1}{8}$ & $\left(\frac{1}{8}\right)^{-D}=27$ \\
\hline$\ldots$ & $\ldots$ & $\ldots$ \\
\hline $2^{r}$ triángulos de lado $\frac{1}{2^{n}}$ & $N=3^{n}, R=\frac{1}{2^{n}}$ & $\left(\frac{1}{2^{n}}\right)^{-D}=3^{n}$ \\
\hline
\end{tabular}


Despejando D obtenemos:

$$
\begin{aligned}
D \ln 2^{n} & =\ln 3^{n} \\
D & =\frac{\ln 3}{\ln 2} \\
D & =1.58496 \ldots
\end{aligned}
$$

¡Una dimensión no entera! Así la dimensión de autosimilaridad $\mathbf{D}$ de un objeto, hecho de $\mathrm{N}$ copias exactas a él mismo y reducidas en un factor R, es:

$$
D=-\frac{\ln N}{\ln R}
$$

Para la línea:

$$
\begin{aligned}
& D=-\frac{\ln 2}{\ln \frac{1}{2}} \\
& D=1
\end{aligned}
$$

Para un cuadrado:

$$
\begin{aligned}
& D=-\frac{\ln 4}{\ln \frac{1}{2}} \\
& D=2
\end{aligned}
$$

Para un cubo:

$$
\begin{aligned}
& D=-\frac{\ln 8}{\ln \frac{1}{2}} \\
& D=3
\end{aligned}
$$

Y para el triángulo de Sierpinski:

$$
\begin{aligned}
& D=-\frac{\ln 3}{\ln \frac{1}{2}} \\
& D=1.58496 \ldots
\end{aligned}
$$

Esparcido por la literatura científica, el concepto de dimensión fractal se utiliza indiscriminadamente para designar dimensión de auto-similaridad (self-similarity dimension), dimensión de capacidad (capacity dimension), dimensión de correlación (correlation dimension), dimensón de información (information dimension ), dimensión de Lyapunov, dimensión de Minkowski-Bouligand y un largo etc.

No vamos a detallar todas las definiciones y diferencias entre todos los conceptos de dimensión. Pero sí creemos necesario ocuparnos de los más usados. Antes de abordar otras definiciones nos detendremos, en la siguiente sección, en algunas de implicaciones matemáticas de la auto-similaridad.

En las primeras secciones de este capítulo hemos visto como al medir la longitud, el área o el volumen de objetos geométricos, el valor medido dependía de la resolución utilizada, del objeto que empleábamos para recubrir nuestra figura. Denominamos relación de escala a la forma matemática que describe esta relación funcional entre lo medido y la resolución de medida. Veamos cómo la autosimilaridad determina la relación de escala. La autosimilaridad se expresa matemáticamente de la siguiente manera. Si a cierta escala de resolución $r$ nuestra medida es $P(r)$, entonces a escala $(a r)$ nuestra medida $P($ ar $)$ será proporcional a $P(r)$. Es decir,

$$
P(\text { ar })=k P(r)
$$


donde $k$ es una constante de proporcionalidad. ¿Cómo dependerá entonces $P$ de $r$, la medida de la resolución?¿Qué relaciones matemáticas son compatibles con la autosimilaridad?

La relación de escala más conocida es la ley de potencias (power law) que se expresa como:

$$
P(r)=c r^{\mu}
$$

Demostrarlo es fácil:

$$
P(a r)=c a^{\mu} r^{\mu}
$$

y haciendo $k=c a^{\mu}$ recuperamos la autosimilaridad:

$$
P(r)=k r^{\mu}
$$

Existe una condición más general denominada forma completa (full form), que incluye como caso particular la ley de potencias:

$$
P(r)=c r^{\mu} f\left(\frac{\ln r}{\ln b}\right)
$$

con $c, \mu$ y $b$ constantes y $f(x)$ una función periódica, tal que $f(x+1)=f(x)$.

Podemos, de hecho es un método estándar, determinar la dimensión fractal a partir de las relaciones de escala. Es el método más usado a partir de medidas experimentales. En general, el número de piezas N(r) para recubrir una figura geométrica es proporcional a r-D como vimos en la sección anterior:

$$
N(r)=r^{-D}
$$

Supongamos que sobre nuestra figura geométrica hemos hecho medidas de longitud, área, volumen u otra medida $P(r)$, que como hemos dicho depende de nuestra escala de medida $r$. Cada uno de los $N(r)$ objetos que hemos empleado para recubrir la figura contribuye a la medida final con $r^{d}$, donde $d$ es igual a 1 si se trata de rectas, 2 si de superficies, etc ... Así la medida en función de $r$ será:

$$
\begin{aligned}
P(r) & =N(r) r^{d} \\
& =r^{-D} r^{d} \\
& =r^{d-D}
\end{aligned}
$$

que claramente es una ley de potencias.

En los dos últimos capítulos del curso (Fractales en la Naturaleza y Criticalidad Auto-organizada) exploraremos con mayor detalle las leyes de potencias.

Olvamos de nuevo al concepto de dimensión. Como comentamos, existe todo un repertorio de dimensiones. Conceptualemente cada una determina una propiedad distinta del objeto geométrico sobre el que la medimos. Podemos hacer tres grandes grupos: 
1. Dimensión fractal (p.ej. dimensión de autosimilaridad, de capacidad o de Hausdorff): se refieren a como el objeto geométrico llena el espacio en el que está inmerso. Las dimensiones fractales pueden ser enteras o fraccionarias.

2. Dimensión topológica (p.ej.: dimensión de recubrimiento o iterativa): nos hablan de la conectividad de los puntos del objeto de medida. Nos dice si nuestro objeto es una arista, un plano, un volumen, un hipervolumen, etc. Su valor es siempre entero.

3. Dimensión de inmersión (Embedding dimension)3. : se refiere al espacio que contiene al objeto de estudio. Puede ser de nuevo entera o fraccionaria.

Como vimos la dimensión fractal de autosimilaridad es consistente con nuestra idea intuitiva de dimensión, pues nos proporcionaba 1, 2 y 3 para un segmento, un cuadrado y un cubo respectivamente.

Una definición más general de dimensión fractal que la de autosimilaridad nos la proporciona la llamada dimensión de capacidad (capacity dimension). Para poder medir la dimensión de autosimilaridad necesitamos que nuestro objeto sea perfectamente autosimilar. Podemos relajar esta condición y utilizar el mismo método de recubrimiento para medir la dimensión de capacidad. Recubriremos, independientemente de la figura geométrica a estudio, con "bolas" de radio $r$. Determinamos el mínimo número $N(r)$ de bolas de tamaño $r$ que necesitamos para recubrir completamente al conjunto de puntos que forma nuestra figura. $\mathrm{La}$ dimensión de capacidad es:

$$
D=\lim _{r \rightarrow 0} \frac{\ln N(r)}{\ln \frac{1}{r}}
$$

es decir un cociente que nos resulta familiar, pero haciendo el radio cero. La dimensión de capacidad es así una generalización de la dimensión de autosimilaridad y ambas coinciden para objetos totalmente autosimilares."

En esa cita textual se aprecian varias definiciones de dimensión. A veces los proceso no lineales generan extraños fenómenos, como el principio de sincronización que explica magistralmente el profesor Steven Strogat en: http://www.ted.com/talks/steven_strogatz_on_sync.html

A quienes quieran leer el famoso libro de Mandelbrot "The Fractal Geometry of Nature" puden bajarlo del sitio: http://www.4shared.com/document/j5qrCWFe/The_Fractal_Geometry_of_Nature.htmlLa BBC editó una serie en que mezcla los conceptos de fractales y caos, el documental se llama la vida secreta del caos, con subtítulos en español http://www · youtube.com/watch?v=hbW_kNp8Wos\&feature=related

El documental se divide para efectos de youtube en siete partes, En la parte 6 http://www. youtube.com/ watch?v=ZalffH3ZdOY\&feature=related se estudia la función compleja , un número complejo, fijando un e iterando esta función consigo misma se obtienen los gráficos de diversos conjuntos fractales. Para diversos valores de y diferente número de iteraciones se obtienen los diversos gráficos, incluyendo el célebre conjunto de Mandelbrot: 


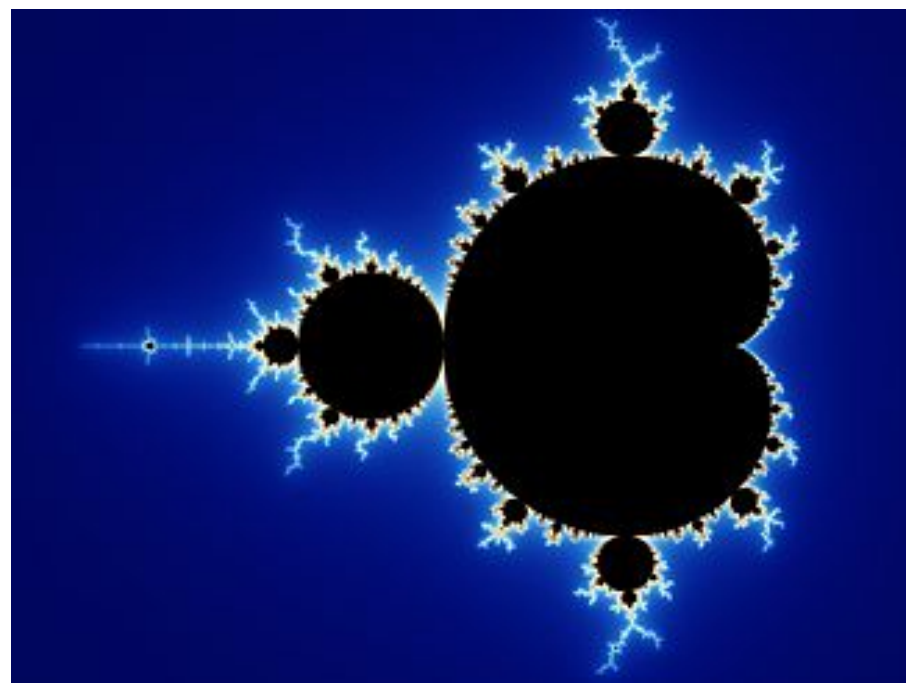

Pocas veces en la historia se tiene la posibilidad de conocer tan de cerca a uno de los actores principales, en el caso de Benoit Mandelbrot tenemos la suerte de tener videos y libros. En campos magnéticos en una escala cuántica se han observado procesos fractales como se reporta en febrero del 2011 en: http: //fractalfoundation.org/category/natural-fractals/

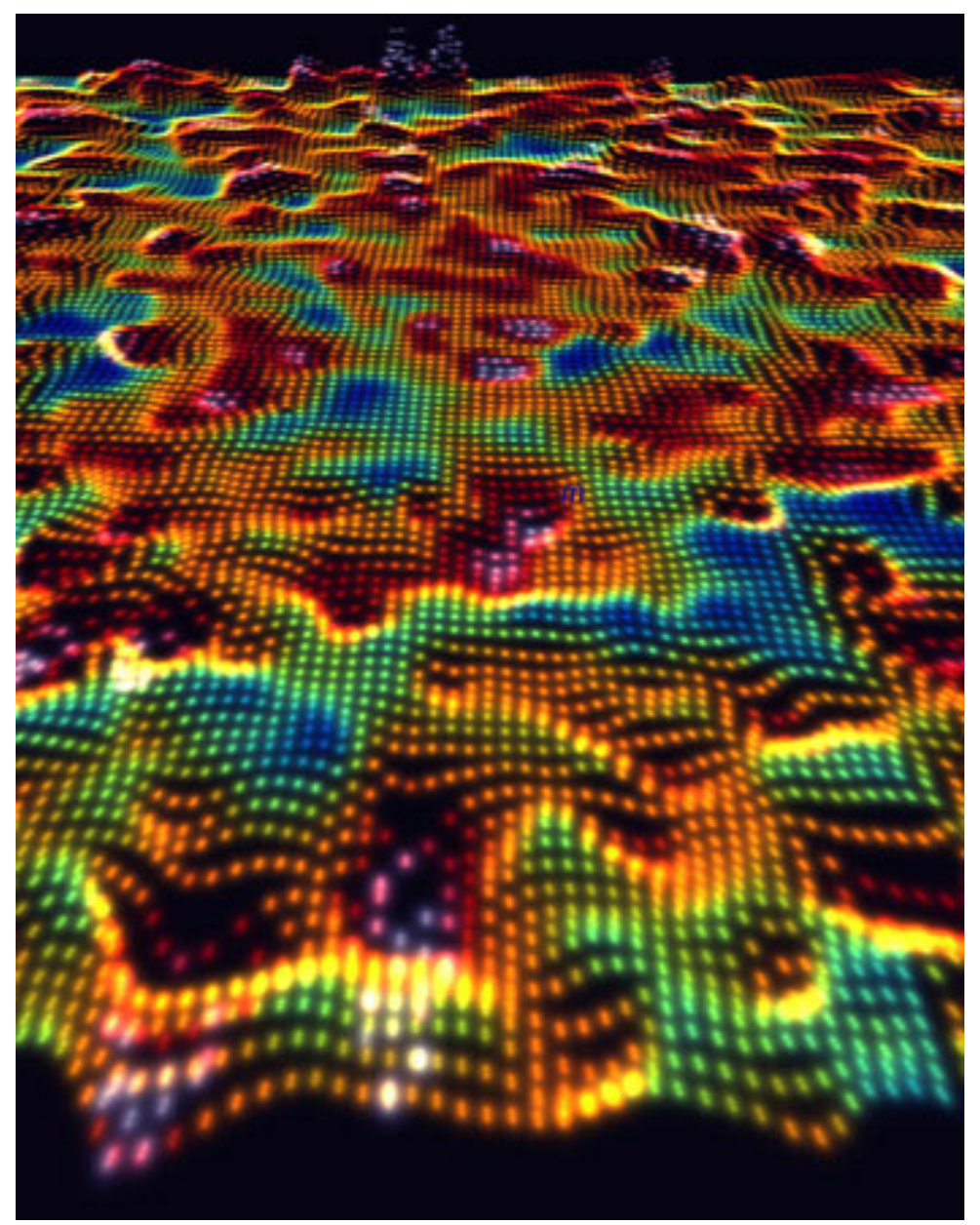


Si los fractales y la teoría del caos nos acercan a una mejor comprensión de los seres vivos está por verse, los indicios son muy esperanzadores, pero indudablemente es una corriente de aire fresco en el vetusto edificio universo de las matemáticas sobre todo en el piso de la geometría. 